\title{
The Frequency of Extrinsic and Intrinsic S Stars in the Henize Sample
}

\author{
SOPHIE VAN ECK ${ }^{1}$, ALAIN JORISSEN ${ }^{1}$, \\ MICHEL MAYOR ${ }^{2}$, STEPHANE UDRY ${ }^{2}$, \\ and MICHEL BURNET ${ }^{2}$
}

${ }^{1}$ Institut d'Astronomie et d'Astrophysique

Université Libre de Bruxelles, Belgium

${ }^{2}$ Observatoire de Genève, Sauverny, Switzerland

Previous studies have identified two distinct families among S stars: intrinsic $\mathrm{S}$ stars exhibiting Tc lines in their spectrum, and extrinsic $\mathrm{S}$ stars lacking Tc lines. Extrinsic S stars were found to be binaries, and probably owe their chemical peculiarities to mass transfer in the binary system. On the contrary, intrinsic S stars are thermally-pulsing AGB stars where the third dredge-up brought heavy elements to the surface. The Henize sample of $205 \mathrm{~S}$ stars south of declination $-25^{\circ}$ is especially well suited for inferring the relative frequency of extrinsic/intrinsic S stars, since it is not biased towards low galactic latitudes where intrinsic $\mathrm{S}$ stars tend to concentrate. Each star has been measured 3 or 4 times over a period of 3 years with the spectrovelocimeter CORAVEL. The search for binaries is complicated by the fact that Mira-type pulsations are frequent among intrinsic S stars. Fortunately, radial-velocity variations due to atmospheric motions are generally associated with very broad and asymmetric CORAVEL cross-correlation profiles. Furthermore, such a criterion based on the CORAVEL line-width index $S b$ (which reduces to a luminosity indicator, since it is found to increase with $M_{\text {bol }}$ ) correlates very well with the Tc/no-Tc dichotomy (as derived from high-resolution spectroscopy for the brightest stars of the sample). It thus provides an independent way to distinguish extrinsic $\left(S b<5 \mathrm{~km} \mathrm{~s}^{-1}\right)$ from intrinsic $\left(S b \geq 5 \mathrm{~km} \mathrm{~s}^{-1}\right) \mathrm{S}$ stars. The frequency of extrinsic S stars is found to be $38 \pm 5 \%$. The distribution of the standard deviation of the radial velocity for extrinsic stars is flatter than that of intrinsic stars, reflecting the large frequency of binaries among extrinsic stars. The galactic distribution of the Henize sample can be described with a scale height above the galactic plane of $180 \mathrm{pc}$ for intrinsic stars compared to $580 \mathrm{pc}$ for the whole Henize sample (assuming $M_{V}=-2$ for intrinsic and $M_{V}=-1$ for extrinsic S stars). 